\title{
Collagen peptide ingestion alters lipid metabolism-related gene expression and the unfolded protein response in mouse liver
}

\author{
Chisa Tometsuka ${ }^{1}$, Yoh-ichi Koyama ${ }^{1}$, Tomoko Ishijima ${ }^{2}$, Tsudoi Toyoda ${ }^{2}$, Miki Teranishi ${ }^{3}$, \\ Kazushige Takehana ${ }^{3}$, Keiko Abe ${ }^{2,4}$ and Yuji Nakai ${ }^{2,5 *}$ \\ ${ }^{1}$ Research Institute of Biomatrix, Nippi Inc., 520-11 Kuwabara, Toride, Ibaraki 302-0017, Japan \\ ${ }^{2}$ Graduate School of Agricultural and Life Sciences, The University of Tokyo, 1-1-1 Yayoi, Bunkyo-ku, Tokyo 113-8657, Japan \\ ${ }^{3}$ Department of Veterinary Microanatomy, School of Veterinary Medicine, Rakuno Gakuen University, 582 Bunkyodai- \\ Midorimachi, Ebetsu, Hokkaido 069-8601, Japan \\ ${ }^{4}$ Project on Health and Anti-aging, Kanagawa Academy of Science and Technology, Life Science and Environment Research \\ Center 4F C-4, 3-25-13 Tonomachi, Kawasaki-ku, Kawasaki, Kanagawa 210-0821, Japan \\ ${ }^{5}$ Institute for Food Sciences, Hirosaki University, 2-1-1 Yanagawa, Aomori, Aomori 038-0012, Japan \\ (Submitted 6 June 2016 - Final revision received 29 November 2016 - Accepted 29 November 2016 - First published online 16 January 2017)
}

\section{Abstract}

Ingestion of collagen peptide (CP) elicits beneficial effects on the body, including improvement in blood lipid profiles, but the underlying mechanisms remain unclear. The purpose of this study was to investigate the effects of $\mathrm{CP}$ ingestion on the liver, which controls lipid metabolism in the body. Male $\mathrm{BALB} / \mathrm{CCrSlc}$ mice were bred with the AIN-93M diet containing $14 \%$ casein or the AIN-93M-based low-protein diet containing $10 \%$ casein or a diet containing $6 \%$ casein $+4 \% \mathrm{CP}$ for 10 weeks ( $n$ 12/group). Total, free and esterified cholesterol levels in the blood decreased in the CP group. DNA microarray analysis of the liver revealed that expressions of genes related to lipid metabolic processes such as the PPAR signalling pathway and fatty acid metabolism increased in the CP group compared with the $10 \%$ casein group. The expressions of several genes involved in steroid metabolic process, including Cyp7a1 and Cyp8b1, were decreased, despite being targets of transcriptional regulation by PPAR. These data suggest that lipid metabolism in the liver is altered by CP ingestion, and the decrease in blood cholesterol levels in the CP group is not due to enhancement of the steroid metabolic process. On the other hand, expressions of genes related to the unfolded protein response (UPR) significantly decreased at the mRNA level, suggesting that CP ingestion lowers endoplasmic reticulum stress. Indeed, protein levels of phosphorylated inositol-requiring enzyme 1 decreased after $\mathrm{CP}$ ingestion. Taken together, $\mathrm{CP}$ affects the broader pathways in the liver - not only lipid metabolism but also UPR.

Key words: Collagen peptide: Liver: Lipid metabolism: Unfolded protein response: DNA microarray

Collagen is the most abundant protein in the body, comprising about one-third of total proteins. Collagen is contained in typical diets consisting of animal products such as meat, bone, tendon or fish $\operatorname{skin}^{(1)}$. Collagen extracted from collagen-rich materials with hot water is known as gelatin. Gelatin and its partial hydrolysate (collagen peptide (CP)) are widely used as food supplements. Ingestion of collagen has several beneficial effects, including increased bone mineral density ${ }^{(2,3)}$, muscle strength ${ }^{(4)}$, attenuation of joint pain and disability ${ }^{(5,6)}$, as well as increased diameter and density of collagen fibrils in the tendon and $\operatorname{skin}^{(7,8)}$.

The beneficial effects of collagen ingestion are not restricted to collagen-rich tissues such as the skin, bone or tendon. Collagen ingestion suppresses the activity of angiotensin I-converting enzyme, resulting in decreased blood pressure $^{(9,10)}$. Ingestion of collagen also reportedly modulates blood lipid profiles in rats ${ }^{(3,11,12,14)}$, mice ${ }^{(13)}$ and rabbits ${ }^{(15)}$; TAG and/or total cholesterol levels are markedly reduced in these animals when fed a CP-containing diet. It has been expected that the ingestion of collagen, which has been shown to elicit effects in various parts of the body ${ }^{(2-15)}$, likely affects the liver, which is involved in various biological functions including lipid metabolism, detoxification, protein synthesis, hormone production and production of biochemicals necessary for digestion ${ }^{(16)}$. However, there are a few reports on the effects of collagen ingestion in the liver. In addition, even though the liver is involved in various biological functions, the condition of the liver is difficult to assess visually, unlike the skin or joints. Therefore, we focused on the effects of collagen ingestion on liver gene expression, and attempted to investigate this using a nutrigenomic approach using DNA microarray.

Abbreviations: $6 \mathrm{C}+4 \mathrm{CP}$, AIN-93M-based low-protein diet containing $6 \%$ protein (as casein) plus 4\% protein (as collagen peptide); 10C, AIN-93M-based low-protein diet containing $10 \%$ protein (as casein); cDNA, complementary DNA; CP, collagen peptide; GO, Gene Ontology; IRE1, inositol-requiring enzyme 1; p-IRE1, phospho-IRE1.

* Corresponding author: Y. Nakai, fax +81 17781 7071, email yunakai@hirosaki-u.ac.jp 
We have previously reported the effect of collagen ingestion on bone mineral density in mice ${ }^{(2)}$. In that study, the four diet conditions of $14 \%$ casein, $10 \%$ casein, $6 \%$ casein $+8 \%$ gelatin, and $6 \%$ casein $+4 \%$ gelatin were compared to investigate the effect of collagen ingestion under conditions of normal protein ratio diet (14\%) or moderately low-protein diet (10\%). Significant in vivo effects of gelatin ingestion were observed; mice fed a $6 \%$ casein $+4 \%$ gelatin diet exhibited significantly higher bone mineral density than those fed a $10 \%$ casein diet. In the present study, we used similar diets containing $10 \%$ casein and $6 \%$ casein $+4 \%$ CP (fish derived, MW 4000-6000, fragments of amino acid sequences of collagen that consist of - Gly-X-Y triplets; the $\mathrm{X}$ and $\mathrm{Y}$ positions are frequently occupied by proline and hydroxyproline, respectively) to examine how collagen ingestion modulates gene expression in the liver, as determined by DNA microarray analysis. We also used the $14 \%$ casein diet to confirm the biological state under the condition of normal protein ratio diet.

\section{Methods}

\section{Animals and diets}

Male 6-week-old BALB/cCrSlc mice were purchased from Japan SLC, Inc., and were acclimated to breeding conditions for 1 week in the laboratory animal facilities of Rakuno Gakuen University. The animals were divided into three groups ( $n 12$ each) on the basis of body weight, so that the average body weight was equivalent among groups. Mice were housed individually under conventional conditions $\left(20 \pm 2^{\circ} \mathrm{C}, 12 \mathrm{~h}\right.$ light $-12 \mathrm{~h}$ dark cycle) with free access to water and food. The basic powder diet AIN-93M containing $14 \%$ protein (as casein) (14C) and the AIN-93M-based low-protein diets containing $10 \%$ protein (as casein) (10C) or $6 \%$ protein (as casein) plus $4 \%$ protein (as CP, fish derived, MW 4000-6000; Nippi Inc.) $(6 \mathrm{C}+4 \mathrm{CP})$ were prepared by Oriental Yeast Co., Ltd (Table 1). Maize starch was used to compensate for the decreased weight of protein in the low-protein diets. Body weight as well as food and water intakes were measured once per week.
After 10 weeks of rearing on the test diets, sampling was performed in the morning after a 3-h fasting period. After anaesthesia with $50 \mathrm{mg} / \mathrm{kg}$ sodium pentobarbital, blood was sampled from the right ventricle with a heparinised syringe, and plasma was stored at $-80^{\circ} \mathrm{C}$. For DNA microarray and Western blotting analyses, three mice exhibiting around median food efficiency (weight gain/food intake) were selected from each group. The whole liver was removed, and a portion of the first lobe (about $5 \times 5 \times 3 \mathrm{~mm}$ ) was stored in RNAlater ${ }^{\circledR}$ (Ambion; Thermo Fisher) before isolation of total RNA. The residual liver was snap-frozen in $\mathrm{N}_{2}$ and stored at $-80^{\circ} \mathrm{C}$ until used for biochemical analyses.

This study was conducted in accordance with the Act on Welfare and Management of Animals in Japan (Act No. 105 of 1 October 1973; partially revised on 22 June 2005), the Standards Relating to the Care and Management of Laboratory Animals and Relief of Pain (Notice No. 88 of the Ministry of the Environment, dated 28 April 2006), and the Guidelines for Methods of Animal Disposition Procedures (Prime Minister's Office Notification No. 40, dated 4 July 1995; partially revised on 12 November 2007: Notice No. 105 of the Ministry of the Environment). The study protocol was approved by the Ethics Committee of Rakuno Gakuen University (No. VH23A8). We have read Animal Research: Reporting of In Vivo Experiments (ARRIVE) guidelines and confirmed that our experiments complied with these guidelines. The animal model and numbers in our study were decided after referring to other previous studies ${ }^{(2,13)}$. In addition, the animal experiments were conducted in a blinded manner.

\section{Biochemical analysis of blood}

Biochemical analysis of blood plasma samples from all animals was entrusted to Nagahama Life Science Laboratory, Oriental Yeast Co., Ltd. The routine biochemical analyses were performed by absorbance measurements based on enzymatic methods using 7180 Clinical Analyzer (Hitachi High-Technologies Corporation). The panel of tests included the following: total cholesterol (T-CHO), free cholesterol (F-CHO), total protein, albumin (ALB), aspartate aminotransferase (AST; GOT), alanine aminotransferase (ALT; GPT), alkaline phosphatase, lactate dehydrogenase, leucine

Table 1. Diet composition(\%)

\begin{tabular}{|c|c|c|c|}
\hline Ingredients & $14 \%$ casein diet $(14 \mathrm{C})$ & $10 \%$ casein diet $(10 \mathrm{C})$ & Collagen diet $(6 \mathrm{C}+4 \mathrm{CP})$ \\
\hline Casein $\left(85.1 \%{ }^{\star}\right)$ & $16.45(14.01) \dagger$ & $11.75(10.00) \dagger$ & $7.05(6.00) \dagger$ \\
\hline Collagen peptide $\left(94.3 \% \%^{*}\right)$ & 0.00 & 0.00 & $4.24(4.00) \dagger$ \\
\hline Maize starch & $44 \cdot 12$ & $47 \cdot 82$ & $48 \cdot 28$ \\
\hline Pre-gelatinisation maize starch & $15 \cdot 50$ & $16 \cdot 50$ & $16 \cdot 50$ \\
\hline Sucrose & $10 \cdot 00$ & $10 \cdot 00$ & $10 \cdot 00$ \\
\hline Soyabean oil & 4.00 & 4.00 & 4.00 \\
\hline Cellulose & $5 \cdot 00$ & $5 \cdot 00$ & $5 \cdot 00$ \\
\hline Mineral mixture & 3.50 & 3.50 & 3.50 \\
\hline Vitamin mixture & 1.00 & 1.00 & 1.00 \\
\hline L-Cystine & $0 \cdot 18$ & $0 \cdot 18$ & $0 \cdot 18$ \\
\hline Choline bitartrate & 0.25 & 0.25 & 0.25 \\
\hline$t$-Butylhydroquinone & Trace & Trace & Trace \\
\hline Total & 100 & 100 & 100 \\
\hline
\end{tabular}

* Protein content of milk casein or collagen peptide.

$\dagger$ Values in parentheses indicate protein content of milk casein or collagen peptide in each diet. $\neq 0.0008 \%$. 
aminopeptidase, $\gamma$-glutamyl transferase, choline esterase, TAG, LDL-cholesterol, HDL-cholesterol, total bilirubin, total bile acid, pyruvic acid, total ketone bodies and glucose. The data on esterified cholesterol (E-CHO) were estimated by the calculation $(\mathrm{T}-\mathrm{CHO}=\mathrm{F}-\mathrm{CHO}+\mathrm{E}-\mathrm{CHO})$. In addition, the following items were measured by ELISA: adiponectin, insulin, leptin and insulin-like growth factor-1 (IGF-1).

\section{Measurement of liver lipids}

Determination of TAG as well as total and free cholesterol levels in liver samples from all animals was entrusted to Skylight Biotech, Inc. The lipid components of the liver samples were extracted using chloroform-methanol mixture solution, and determined by absorbance measurements based on enzymatic methods (reagent kits: Cholestest ${ }^{\circledR} \mathrm{TG}$ or Cholestest ${ }^{\circledR} \mathrm{CHO}$; Sekisui Medical Co., Ltd and Cholescolor ${ }^{\circledR}$ Liquid FC; Toyobo Co., Ltd).

\section{RNA preparation}

Total RNA was isolated from each liver sample using ISOGEN ${ }^{\circledR}$ reagent (Nippon Gene Co.) and subsequently purified using an RNeasy ${ }^{\circledR}$ Mini Kit (Qiagen K.K.), according to the manufacturer's protocol. Total RNA quality and quantity were assessed by agarose gel electrophoresis and spectrophotometry. Total RNA was used for the DNA microarray assay, as described below.

\section{DNA microarray experiment}

DNA microarray analysis was performed on liver samples from a total of six mice (three mice each from the $10 \mathrm{C}$ and $6 \mathrm{C}+4 \mathrm{CP}$ groups) by choosing average individuals from each group on the basis of their food efficiency values. For each sample, double-stranded complementary DNA (cDNA) was synthesised from $100 \mathrm{ng}$ of purified total RNA, and biotinylated cRNA was transcribed from the cDNA using a GeneChip ${ }^{\circledR}$ 3' IVT Express Kit (Affymetrix). The cRNA were subsequently fragmented and hybridised to a GeneChip ${ }^{\circledR}$ Mouse Genome 430 2.0 Array (Affymetrix) containing over 34000 mouse genes. The arrays were washed and labelled with streptavidin-phycoerythrin using a GeneChip ${ }^{\circledR}$ Hybridization, Wash and Stain Kit and Fluidics Station 450 system (Affymetrix). Fluorescence was detected using a GeneChip ${ }^{\circledR}$ Scanner 3000 7G (Affymetrix). All experimental procedures were carried out according to the manufacturer's instructions. All microarray data were Minimum Information About a Microarray Experiment (MIAME) compliant, and were deposited in a MIAME-compliant database - the National Center for Biotechnology Information Gene Expression Omnibus (http:// www.ncbi.nlm.nih.gov/geo/; GEO Series accession number GSE75171) - as detailed on The Functional Genomics Data Society website (http://fged.org/projects/miame/).

\section{DNA microarray data analysis}

Affymetrix GeneChip ${ }^{\circledR}$ Command Console software was used to reduce the array images to the intensity of each probe (CEL files). The CEL files were quantified using the Factor Analysis for Robust Microarray Summarization algorithm ${ }^{(17)}$ with the statistical packages $\mathrm{R}^{(18)}$ and Bioconductor ${ }^{(19)}$. Probe sets found to be differentially expressed between the $10 \mathrm{C}$ and the $6 \mathrm{C}+4 \mathrm{CP}$ groups were identified according to the Rank products method ${ }^{(20)}$ using the statistical package R. The annotation file for the Mouse Genome 4302.0 Array was downloaded from the Affymetrix website (30 March 2016, http://www.affymetrix.com/). Probe sets with a false-discovery rate (FDR) $<0.05$ were considered to reflect ingestion of $\mathrm{CP}$. Gene-annotation enrichment analysis was then performed using the web tool Database for Annotation, Visualization, and Integrated Discovery (version 6.7) ${ }^{(21)}$ with Gene Ontology (GO) and Kyoto Encyclopedia of Genes and Genomes (KEGG) annotations. Expression Analysis Systematic Explorer (EASE) scores, which are modified Fisher's exact test $P$-values ${ }^{(22)}$, were used to evaluate statistically over-represented GO terms or KEGG pathways from the differentially expressed genes. Benjamini \& Hochberg ${ }^{(23)}$ FDR corrections for multiple testing were used to correct the results. GO terms with FDR-corrected $P$-values of $<0.05$ were regarded as significantly enriched.

\section{Real-time quantitative RT-PCR analysis}

cDNA synthesis of total RNA ( $2 \mu \mathrm{g} /$ sample) was carried out using High-Capacity cDNA Reverse Transcription Kit (Thermo Fisher-Applied Biosystems) according to the manufacturer's instructions. To perform real-time quantitative PCR, specific primers and probes for mouse PPAR $\alpha$, acyl-CoA oxidase 1, palmitoyl (Acox1), inositol-requiring enzyme 1 (IRE1) and ER degradation enhancer, mannosidase alpha-like 1 (Edem1) (as targets) and actin beta (Actb) as an endogenous control were purchased from Thermo Fisher-Applied Biosystems (TaqMan $^{\circledR}$ Gene Expression Assays; ID: Mm00440939_m1, Mm01246834_m1, Mm00470233_m1, Mm00551797_m1, Mm02619580_g1, respectively). PCR was performed using 7300 Real-time PCR System (Thermo Fisher-Applied Biosystems) following the manufacturer's protocol. The reaction solution for PCR was prepared in volumes of $20 \mu \mathrm{l} /$ well containing $10 \mu \mathrm{l}$ TaqMan ${ }^{\circledR}$ Universal PCR Master Mix (Thermo Fisher-Applied Biosystems), $1 \mu \mathrm{l}$ primer and probe, and $9 \mu \mathrm{l}$ cDNA in RNasefree water (500-fold dilution of the cDNA synthesis solution), and PCR was performed using two wells per sample. Amplification conditions were $2 \mathrm{~min}$ at $50^{\circ} \mathrm{C}, 10 \mathrm{~min}$ at $95^{\circ} \mathrm{C}$ and forty cycles each consisting of $15 \mathrm{~s}$ at $95^{\circ} \mathrm{C}$ and $1 \mathrm{~min}$ at $60^{\circ} \mathrm{C}$. The standard curves were drawn by serial dilutions of the cDNA sample. The $C_{t}$ value obtained by amplification of each target was compared among the samples after normalisation using Actb expression levels.

\section{Western blotting}

Approximately $30 \mathrm{mg}$ of each liver sample was excised on ice and homogenised in Tissue Protein Extraction Reagent (Thermo Fisher Scientific, Inc.) containing a $1 / 100$ volume of Halt Protease and Phosphatase Inhibitor Cocktail (Thermo Fisher Scientific, Inc.). Each sample was then diluted with $2 \times$ sample buffer mix for SDS-PAGE (pH 6.8, 4\% SDS, 25\% glycerol, 125 mm-TRIS-HCl, $0 \cdot 02 \%$ bromophenol blue, $10 \%$ 2-mercaptoethanol). After brief sonication and centrifugation $\left(4^{\circ} \mathrm{C}, 1000\right.$ $\mathrm{rpm}, 10 \mathrm{~min}$ ), the supernatant was collected and boiled at $100^{\circ} \mathrm{C}$ 
for 3 min. Each sample of $10 \mu$ lwas separated on an $8 \%$ SDS-PAGE gel and transferred to a polyvinylidene difluoride membrane (Millipore). The membrane was blocked by incubation in TRIS-PBS (0.1\% Tween 20, PBS) containing 0.3\% skimmed milk for $30 \mathrm{~min}$ at room temperature. After blocking, the membrane was probed with rabbit antibodies against IRE1 or phospho-IRE1 (p-IRE1) (Abcam) and a rabbit antibody against glyceraldehyde-3phosphate dehydrogenase (Cell Signaling Technology, Inc.) at 1:1000 dilution. All primary antibodies were detected using an horseradish peroxidase-linked anti-rabbit IgG antibody (Cell Signaling Technology) and visualised using a Luminescent Image Analyzer LAS-3000 (Fuji Photo Film Co., Ltd). Visualised bands were analysed by densitometry using Multi Gauge version 3.0 (Fuji Photo Film Co.).

\section{Statistical analysis}

Statistical differences between the three groups for all data except those of DNA microarray analyses were assessed by ANOVA with the Tukey-Kramer test, with $P<0.05$ considered indicative of significance. Real-time quantitative RT-PCR analysis was performed for the two groups $(10 \mathrm{C}, 6 \mathrm{C}+4 \mathrm{CP})$ similar to DNA microarray analysis, and the data were compared with Student's $t$ test.

\section{Results}

\section{Dietary intake and growth}

Body weight gain, water intake and food intake data are shown in Table 2 . Food intake was significantly higher in the $6 \mathrm{C}+4 \mathrm{CP}$ group compared with the $14 \mathrm{C}$ and $10 \mathrm{C}$ groups. However, there were no significant differences in body weight gain between groups.

\section{Biochemical analysis of blood}

Biochemical analysis of blood revealed that adiponectin and total, free and esterified cholesterol levels were significantly lower in the $6 \mathrm{C}+4 \mathrm{CP}$ group than in the $10 \mathrm{C}$ group (Fig. 1(A-D)). No significant changes were observed in other parameters including ALB, AST, ALT and TAG (data not shown). These results suggest that ingestion of $\mathrm{CP}$ lowers blood cholesterol levels without causing liver damages.

\section{Liver lipid content}

No significant differences were detected in TAG or total and free cholesterol levels in the liver (data not shown).
Increased expressions of genes involved in lipid metabolism in the liver

The ingestion of $\mathrm{CP}$ resulted in the significant up-regulation of 485 probe sets. Enrichment analysis using GO annotation revealed that the expressions of genes related to fatty acid metabolism, cofactor metabolic processes, coenzyme metabolic processes and acyl-CoA metabolic processes were significantly up-regulated in the $6 \mathrm{C}+4 \mathrm{CP}$ group compared with the $10 \mathrm{C}$ group (Table 3 ). Enrichment analysis of the up-regulated gene set using KEGG annotation indicated that expressions of genes involved in biosynthesis of unsaturated fatty acids, PPAR signalling pathway and fatty acid metabolism were significantly up-regulated in the $6 \mathrm{C}+4 \mathrm{CP}$ group (Table 5). Notably, $\operatorname{PPAR} \alpha$, one of the subtypes of PPAR, was up-regulated by $\mathrm{CP}$ ingestion. The significantly up-regulated genes related to the PPAR $\alpha$ signalling pathway are summarised in Fig. 2(a). Among the up-regulated PPAR $\alpha$ target genes involved in lipid metabolism were genes related to lipogenesis malic enzyme 1, NADP+-dependent, cytosolic (Me1), stearoyl-CoA desaturase 1 (Scd1), fatty acid desaturase 2 (Fads2), fatty acid $\beta$-oxidation (Acox1, carnitine palmitoyltransferase 1a, liver (Cpt1a), enoyl-CoA, hydratase/3-hydroxyacyl CoA dehydrogenase (Ehhadh), acetyl-CoA acyltransferase 1A (Acaa1a), cytochrome P450, family 4, subfamily a, polypeptide 10 (Cyp4a10), Cyp4a14) and fatty acid transport acyl-CoA synthetase long-chain family member 1 (Acsl1).

\section{Decreased expression of genes involved in the unfolded protein response}

The ingestion of $\mathrm{CP}$ resulted in the significant down-regulation of 395 probe sets. Enrichment analysis using GO annotation revealed that the expressions of genes related to steroid metabolic process, response to unfolded protein and response to protein stimulus were down-regulated by $\mathrm{CP}$ ingestion (Table 4). In contrast, no significantly enriched KEGG pathways were found in the down-regulated gene set. Down-regulated genes included in the 'response to unfolded protein' GO term are summarised in Fig. 2(b). In the $6 \mathrm{C}+4 \mathrm{CP}$ group, expressions of the gene encoding IRE1 (also known as Ern1; endoplasmic reticulum to nucleus signaling 1) and its target genes related to the degradation of unfolded proteins Der1-like domain family, member 2 (Derl2), Edem1, synovial apoptosis inhibitor 1, synoviolin (Syun1), chaperon functions DnaJ (Hsp40) homolog, subfamily C, member 3 (Dnajc3) and endoplasmic reticulum (ER) stress-induced cell death mesencephalic astrocyte-derived neurotrophic factor (Manf) were down-regulated.

Table 2. Body weight and food and water intakes (Numbers; mean values with their standard errors)

\begin{tabular}{|c|c|c|c|c|c|c|c|}
\hline & \multirow[b]{2}{*}{$n$} & \multicolumn{2}{|c|}{ Body weight gain (g) } & \multicolumn{2}{|c|}{ Total food intake $(\mathrm{g})$} & \multicolumn{2}{|c|}{ Total water intake $(\mathrm{ml})$} \\
\hline & & Mean & SE & Mean & SE & Mean & SE \\
\hline $14 \%$ casein diet $(14 C)$ & 12 & 5.4 & 0.4 & $211 \cdot 4^{\mathrm{a}}$ & $3 \cdot 2$ & $231 \cdot 2$ & 9.9 \\
\hline $10 \%$ casein diet $(10 \mathrm{C})$ & 12 & $5 \cdot 2$ & 0.5 & $208 \cdot 4^{\mathrm{a}}$ & $2 \cdot 7$ & $222 \cdot 2$ & 11.7 \\
\hline Collagen diet $(6 \mathrm{C}+4 \mathrm{CP})$ & 12 & 5.5 & 0.3 & $229 \cdot 1^{\mathrm{b}}$ & $6 \cdot 1$ & $267 \cdot 6$ & 18.8 \\
\hline
\end{tabular}

a,b Mean values with unlike superscript letters were significantly different $(P<0.05)$. 
(A)

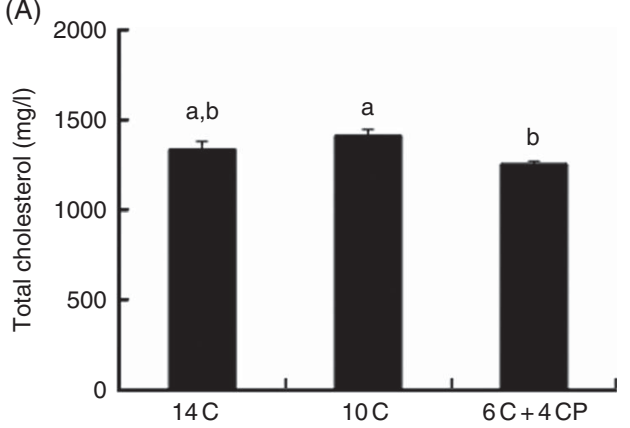

(C)

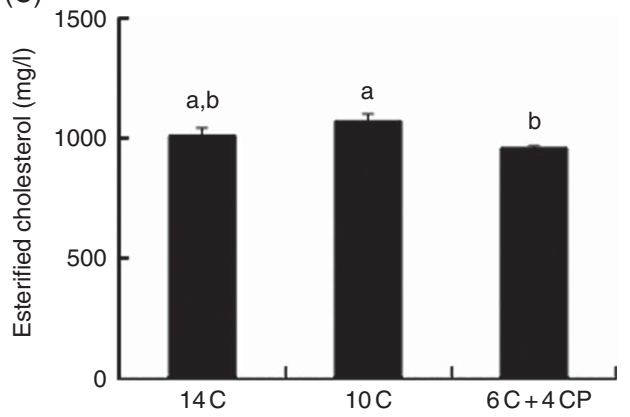

(B)

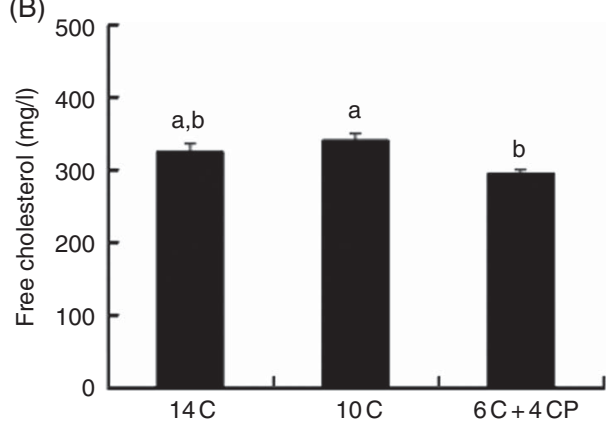

(D)

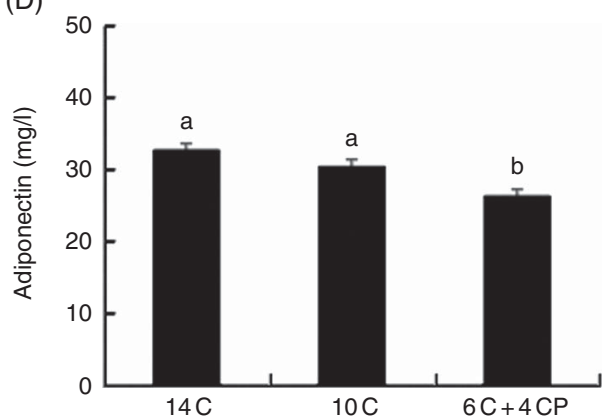

Fig. 1. Blood biochemical parameters. Plasma concentrations of total cholesterol (A), free cholesterol (B), esterified cholesterol (C) and adiponectin (D) are shown. Values are means ( $n 12$ for each group), with standard errors represented by vertical bars. 14C, AIN-93M containing $14 \%$ protein (as casein); $10 \mathrm{C}$, AIN-93M-based low-protein diet containing $10 \%$ protein (as casein); $6 \mathrm{C}+4 \mathrm{CP}$, AIN-93M-based low-protein diet containing $6 \%$ protein (as casein) plus $4 \%$ protein (as collagen peptide). ${ }^{\mathrm{a}, \mathrm{b}}$ Mean values with unlike letters were significantly different $(P<0.05)$.

\section{Changes in inositol-requiring enzyme 1 and phospho- inositol-requiring enzyme 1 protein expression levels}

The DNA microarray analysis identified two factors (PPAR $\alpha$ and IRE1) as targets of CP ingestion. We performed Western blotting analysis of IRE1 protein because we did not expect a significant change in the unfolded protein response (UPR) following $\mathrm{CP}$ ingestion. As shown in Fig. 3(B), the level of IRE1 was significantly lower in the $6 \mathrm{C}+4 \mathrm{CP}$ group compared with the $14 \mathrm{C}$ group, whereas there was no significant difference compared with the 10C group. However, the level of the active form of IRE1 (p-IRE1) was significantly lower in the $6 \mathrm{C}+4 \mathrm{CP}$ group compared with the other two groups (Fig. 3(C)). The ratio of p-IRE1:IRE1 was also the lowest in the $6 \mathrm{C}+4 \mathrm{CP}$ group (Fig. 3(D)). These results suggest that ingestion of $\mathrm{CP}$ suppresses expression of the IRE1 gene and that the level of its active product ( $\mathrm{p}$-IRE1) declines in the liver following $\mathrm{CP}$ ingestion.

\section{Discussion}

In this study, food intake in the $6 \mathrm{C}+4 \mathrm{CP}$ group increased significantly compared with the $14 \mathrm{C}$ and $10 \mathrm{C}$ groups, indicating that energy intake was greater in the $6 \mathrm{C}+4 \mathrm{CP}$ group than in the other groups. However, there was no significant difference in body weight gain between the three groups. One explanation for this observation is that $\mathrm{CP}$ ingestion is associated with enhanced energy generation, as DNA microarray analysis of the liver revealed that the expression of genes related to fatty acid $\beta$-oxidation were up-regulated in the $6 \mathrm{C}+4 \mathrm{CP}$ group compared with the $10 \mathrm{C}$ group. Incidentally, the ratio of amino acids intake in the $6 \mathrm{C}+4 \mathrm{CP}$ group differed from those in the other two casein groups, owing to the difference in amino acid composition of each protein (casein, CP). However, no significant differences were observed in terms of body weight gain and liver lipid contents between the three groups. In addition, no significant changes in blood biomarkers indicative of pathological conditions were observed in any of the groups. These data suggest that the diet conditions examined this study are not associated with any severe adverse effects attributable to amino acid imbalance, such as growth retardation.

PPAR $\alpha$ is a nuclear receptor that functions as a transcription factor, regulating the expression of genes involved in fatty acid catabolism $^{(24-26)}$. In the present study, the expression of PPAR $\alpha$ and its target genes related to lipogenesis, fatty acid $\beta$-oxidation and fatty acid transport increased significantly after $\mathrm{CP}$ ingestion. This suggests the possibility that $\mathrm{CP}$ ingestion enhances lipid metabolism in the liver through activation of PPAR $\alpha$ signalling, as reported for other natural products ${ }^{(27-31)}$. Moreover, expression of the gene encoding Sirt1, which interacts with and activates PPAR $\alpha$ signalling ${ }^{(32,33)}$, was up-regulated in the $6 \mathrm{C}+4 \mathrm{CP}$ group (rank: 233 of the 485 significantly up-regulated probe sets, online Supplementary Table S1).

The expressions of genes involved in the biosynthesis of unsaturated fatty acids were also up-regulated in the $6 \mathrm{C}+4 \mathrm{CP}$ group, including PPAR $\alpha$ targets such as Acaa1a, Acox1, Scd1 and Fads2 (Table 5). Unsaturated fatty acids conjugated with coenzyme A (i.e. fatty acyl-CoA) are transported into the peroxisome or the mitochondria and metabolised via the $\beta$-oxidation pathwayy ${ }^{(34)}$. As unsaturated fatty acids are also known to be endogenous ligands for $\operatorname{PPARs}^{(24-26,35)}$, 
Table 3. A list of biological process Gene Ontology (GO) terms and their component genes significantly up-regulated (false-discovery rate-corrected $P$-value $<0.05)$ by collagen peptide ingestion

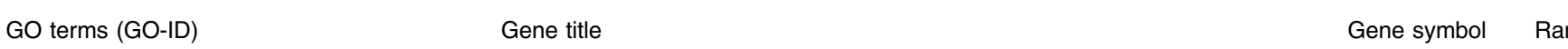

Fatty acid metabolic process (GO:0006631)

Cofactor metabolic process (GO:0051186)

\section{Acyl-CoA thioesterase 3}

Cytochrome P450, family 4, subfamily a, polypeptide 10

Acyl-CoA synthetase long-chain family member 1

Acyl-CoA thioesterase 4

Carnitine acetyltransferase

Acetyl-CoA acyltransferase $1 A$

Enoyl-CoA, hydratase/3-hydroxyacyl CoA dehydrogenase

Stearoyl-CoA desaturase 1

Acyl-CoA oxidase 1, palmitoyl

RIKEN CDNA 9130409123 gene

Hydroxyacyl-CoA dehydrogenase/3-ketoacyl-CoA thiolase/enoyl-CoA

hydratase (trifunctional protein), beta subunit

Carnitine palmitoyltransferase 1a, liver

Fatty acid desaturase 2

Fatty acid desaturase 1

Peroxisome proliferator-activated receptor alpha

Acyl-CoA thioesterase 12

Solute carrier family 27 (fatty acid transporter), member 2

Elongation of very long chain fatty acids (FEN1/Elo2, SUR4/Elo3, yeast)-like 2 Elovl2

Acyl-CoA thioesterase 3

Acyl-CoA thioesterase 2

Acyl-CoA thioesterase 4

Pyruvate dehydrogenase kinase, isoenzyme 4

Hepatic nuclear factor 4, alpha

Enoyl-CoA, hydratase/3-hydroxyacyl CoA dehydrogenase

Carboxylesterase $1 D$

Glutathione S-transferase, theta 2

Aminolevulinic acid synthase 1

Aminolevulinate, delta-, dehydratase

Glutamate-cysteine ligase, catalytic subunit

Nicotinamide phosphoribosyltransferase

Pantothenate kinase 1

Pantothenate kinase 3

Acyl-CoA thioesterase 12

Coproporphyrinogen oxidase

Coenzyme metabolic process (GO:0006732)

Acyl-CoA thioesterase 3

Acyl-CoA thioesterase 2

Acyl-CoA thioesterase 4

Pyruvate dehydrogenase kinase, isoenzyme 4

Hepatic nuclear factor 4, alpha

Enoyl-CoA, hydratase/3-hydroxyacyl CoA dehydrogenase

Carboxylesterase $1 D$

Glutathione S-transferase, theta 2

Glutamate-cysteine ligase, catalytic subunit

Nicotinamide phosphoribosyltransferase

Pantothenate kinase 1

Pantothenate kinase 3

Acyl-CoA thioesterase 12

Acyl-CoA metabolic process (GO:0006637)

Acyl-CoA thioesterase 3

Acyl-CoA thioesterase 2

Acyl-CoA thioesterase 4

Hepatic nuclear factor 4 , alpha

Enoyl-CoA, hydratase/3-hydroxyacyl CoA dehydrogenase

Carboxylesterase $1 D$

Acyl-CoA thioesterase 12

$\begin{array}{lr}\text { Acot3 } & 2 \\ \text { Cyp4a10 } & 7 \\ \text { Acsl1 } & 18 \\ \text { Acot4 } & 21 \\ \text { Crat } & 24 \\ \text { Acaa1a } & 59 \\ \text { Ehhadh } & 66 \\ \text { Scd1 } & 79 \\ \text { Acox1 } & 148 \\ \text { 9130409l23Rik } & 157 \\ \text { Hadhb } & 175 \\ & \\ \text { Cpt1a } & 193 \\ \text { Fads2 } & 277 \\ \text { Fads1 } & 340 \\ \text { Ppara } & 349 \\ \text { Acot12 } & 355 \\ \text { Slc27a2 } & 468 \\ \text { Elovl2 } & 471\end{array}$

Acot3 2

Acot2 13

Acot4 21

Pdk4 22

Hnf4a $\quad 65$

Ehhadh $\quad 66$

Ces1d 76

Gstt2 123

Alas1 135

Alad $\quad 139$

Gclc $\quad 147$

Nampt $\quad 171$

Pank1 325

Pank3 335

Acot12 355

Cpox 364

Acot3

Acot2 13

Acot4 21

Pdk4 22

Hnf4a 65

Ehhadh 66

Ces1d 76

Gstt2 123

Gclc $\quad 147$

Nampt $\quad 171$

Pank1 325

Pank3 335

Acot12 355

Acot3 2

Acot2 13

Acot4 21

Hnf4a $\quad 65$

Ehhadh $\quad 66$

Ces1d 76

Acot12 355

enhancement of this biosynthetic process is predicted to modulate PPAR $\alpha$ signalling and $\beta$-oxidation.

Biochemical analysis of blood samples revealed that total, free and esterified cholesterol levels decreased significantly following $\mathrm{CP}$ ingestion in comparison with casein ingestion (Fig. 1(A-C)). This is consistent with previous studies that examined the lipid-lowering effects of collagen ingestion in humans and animals ${ }^{(3,11-15)}$. In the present study, significant differences were observed neither in the level of blood TAG nor in liver lipid contents, although our DNA microarray data suggest that liver lipid metabolism is enhanced by CP ingestion. Similar results that reduction in blood cholesterol without 
(a)

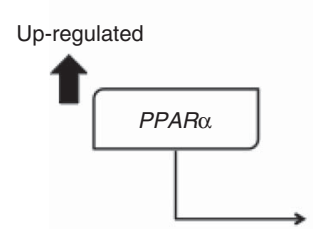

Transcriptional regulation

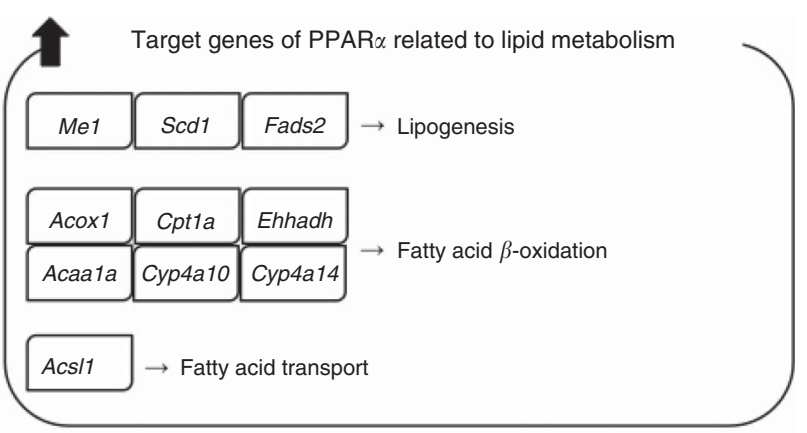

(b)

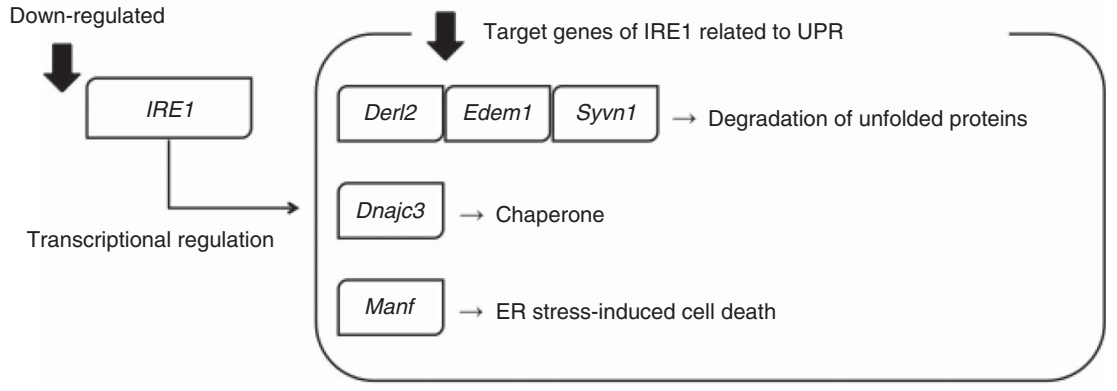

Fig. 2. Two representative biological processes modulated by collagen peptide ingestion. Up-regulated genes related to lipid metabolism, identified by Kyoto Encyclopedia of Genes and Genomes pathway analysis (a), and down-regulated genes related to the unfolded protein response (UPR), identified by Gene Ontology analysis (b) are summarised. IRE1, inositol-requiring enzyme 1; ER, endoplasmic reticulum.

Table 4. A list of biological process Gene Ontology (GO) terms and their component genes significantly down-regulated (false-discovery rate-corrected $P$-value $<0.05)$ by collagen peptide ingestion

\begin{tabular}{|c|c|c|c|}
\hline GO terms (GO-ID) & Gene title & Gene symbol & Rank \\
\hline \multicolumn{4}{|c|}{ Steroid metabolic process (GO:0008202) } \\
\hline & Cytochrome P450, family 7, subfamily a, polypeptide 1 & Сур7а1 & \\
\hline & Peroxisomal biogenesis factor 2 & Pex2 & 66 \\
\hline & High density lipoprotein (HDL) binding protein & Hdllbp & 74 \\
\hline & Serum amyloid $A 1$ & Saa1 & 83 \\
\hline & Farnesyl diphosphate synthetase & Fdps & 130 \\
\hline & ATPase, class I, type $8 B$, member 1 & Atp8b1 & 143 \\
\hline & Short chain dehydrogenase/reductase family $42 E$, member 1 & Sdr42e1 & 158 \\
\hline & Sterol regulatory element binding transcription factor 1 & Srebf1 & 168 \\
\hline & Solute carrier family 37 (glucose-6-phosphate transporter), member 4 & Slc37a4 & 202 \\
\hline & Hydroxy-delta-5-steroid dehydrogenase, 3 beta- and steroid delta-isomerase 3 & $H s d 3 b 3$ & 241 \\
\hline & ATP-binding cassette, sub-family $A(A B C 1)$, member 1 & Abca1 & 245 \\
\hline & Hydroxy-delta-5-steroid dehydrogenase, 3 beta- and steroid delta-isomerase 2 & Hsd3b2 & 275 \\
\hline & Catalase & & 284 \\
\hline & Glucose-6-phosphatase, catalytic & G6pc & 293 \\
\hline \multicolumn{4}{|c|}{ Response to unfolded protein (GO:0006986) } \\
\hline & Synovial apoptosis inhibitor 1 , synoviolin & Syvn1 & \\
\hline & Mesencephalic astrocyte-derived neurotrophic factor & Manf & 13 \\
\hline & DnaJ (Hsp40) homolog, subfamily C, member 3 & Dnajc3 & \\
\hline & Heat shock protein 90, alpha (cytosolic), class $A$ member 1 & Hsp90aa1 & 50 \\
\hline & UBX domain protein 4 & Ubxn4 & 119 \\
\hline & ER degradation enhancer, mannosidase alpha-like 1 & Edem1 & 18 \\
\hline & Endoplasmic reticulum (ER) to nucleus signalling 1 & Ern1 (IRE1) & \\
\hline & Alanyl-tRNA synthetase & Aars & 214 \\
\hline & Heat shock protein 4 like & Hspa4l & \\
\hline & Der1-like domain family, member 2 & Derl2 & \\
\hline \multicolumn{4}{|c|}{ Response to protein stimulus (GO:0051789) } \\
\hline & Synovial apoptosis inhibitor 1, synoviolin & Syvn1 & \\
\hline & Mesencephalic astrocyte-derived neurotrophic factor & Manf & \\
\hline & DnaJ (Hsp40) homolog, subfamily C, member 3 & Dnajc3 & \\
\hline & Heat shock protein 90, alpha (cytosolic), class A member 1 & Hsp90aa1 & \\
\hline & UBX domain protein 4 & Ubxn4 & 119 \\
\hline & ER degradation enhancer, mannosidase alpha-like 1 & Edem1 & \\
\hline & Endoplasmic reticulum (ER) to nucleus signalling 1 & Ern1 (IRE1) & \\
\hline & Alanyl-tRNA synthetase & Aars & \\
\hline & Heat shock protein 4 like & Hspa4l & \\
\hline & Der1-like domain family, member 2 & Derl2 & \\
\hline
\end{tabular}


(A)

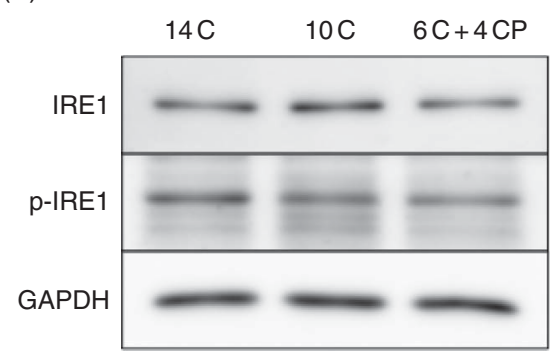

(C)

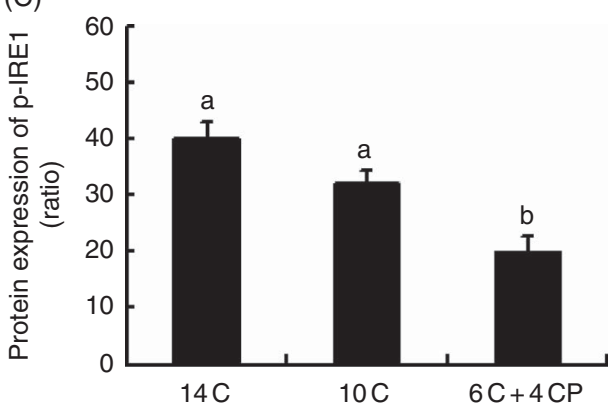

(B)

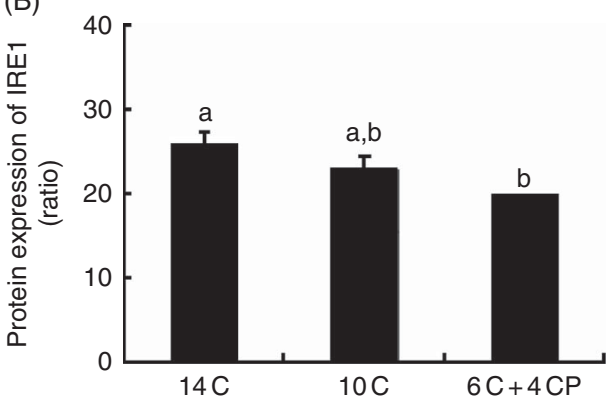

(D)

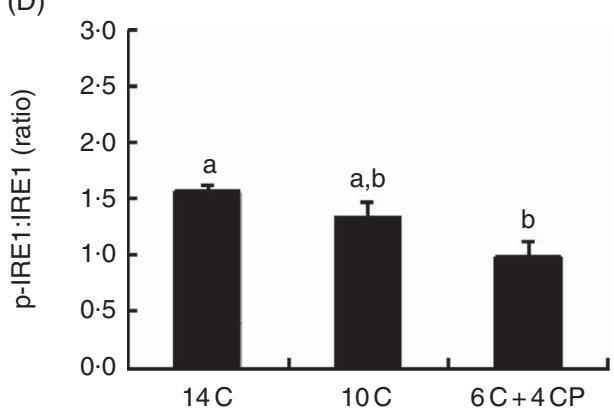

Fig. 3. Western blotting of inositol-requiring enzyme 1 (IRE1) and phosphor-IRE1 (p-IRE1). Western blotting images (A), quantitative data for IRE1 (B) and p-IRE1 (C) protein and $p$-IRE1:IRE1 ratio (D) are shown. The data were obtained from four replicate experiments and are represented as means with their standard errors ( $n 3$ for each group). 14C, AIN-93M containing 14\% protein (as casein); 10C, AIN-93M-based low-protein diet containing 10\% protein (as casein); 6C+4CP, AIN-93M-based low-protein diet containing $6 \%$ protein (as casein) plus $4 \%$ protein (as collagen peptide). The vertical axis (B, C) shows the levels of IRE1 or p-IRE1 protein relative to the level of glyceraldehyde-3-phosphate dehydrogenase (GAPDH) protein. ${ }^{\text {a,b }}$ Mean values with unlike letters were significantly different $(P<0.05)$.

changes in TAG were also observed in our previous study in rats $^{(14)}$. On the other hand, there are several reports that serum TAG is reduced by CP ingestion ${ }^{(11,13)}$, and one explanation for the reduction is described as increase in faecal excretion ${ }^{(13)}$. This discrepancy can be partly accounted for by the differences in the experimental conditions or timing of sampling, but the precise reason is still unclear. However, it is certain that $\mathrm{CP}$ ingestion affects lipid metabolism, and we conceive that PPAR $\alpha$ signalling may be involved in the mechanism of the CP effect. In the $6 \mathrm{C}+4 \mathrm{CP}$ group, the expressions of genes related to primary bile acid biosynthesis were down-regulated (Table 4; steroid metabolic process). It is known that the process of primary bile acid biosynthesis is the major mechanism for the removal of cholesterol from the body, as by synthesising primary bile acid via oxidation of cholesterol. Although PPAR $\alpha$ signalling was up-regulated in the $6 \mathrm{C}+4 \mathrm{CP}$ group (Tables 3 and 5), certain genes of PPAR $\alpha$ targets (Cyp7a1, Cyp8b1, Cyp27a1 and Acox2; related to primary bile acid biosynthesis) were down-regulated. Our data suggest that the decrease in blood cholesterol levels associated with $\mathrm{CP}$ ingestion is not solely due to enhancement of primary bile acid biosynthesis. We presumed that the downregulation of these genes occurred as a reaction of suppressing excess decrease of cholesterol for cholesterol homoeostasis in the body. It has been reported that some proteins other than collagen, such as soyabeans and whey, also have cholesterollowering effects, and the main mechanism of the effects is the suppression of cholesterol absorption (i.e. faecal excretion of cholesterol $)^{(36,37)}$. We presumed that $\mathrm{CP}$ ingestion caused the continuous decrease in internal cholesterol, thus the process of primary bile acid biosynthesis was suppressed. In addition, the bile acids are secreted into the intestine and recirculated to the liver by the enterohepatic circulation; there is a negative-feedback mechanism mediated by the bile acids themselves in the liver, suppressing the expression of the genes related to primary bile acid biosynthesis including Cyp $7 a 1$ and Cyp $8 b 1^{(38)}$. It is possible that this mechanism may be related to our results in this study. To clarify the precise mechanisms involved in the cholesterol-lowering effects of CP ingestion, more detailed investigations should be performed that focus on the absorption, excretion, synthesis and transport of cholesterol in each organ. However, it is a new finding of our study that the effect of blood cholesterol lowering by CP ingestion is not due to enhancement of primary bile acid biosynthesis. In addition, the level of adiponectin in the blood was significantly lower in the $6 \mathrm{C}+4 \mathrm{CP}$ group compared with the other groups. Decreased blood adiponectin levels are associated with the development of various pathological conditions ${ }^{(31,39,40)}$. However, adiponectin levels in the present study were within the normal range for mice $(17 \cdot 0-77 \cdot 0 \mathrm{mg} / \mathrm{l})$, and no obesity or increase in blood/liver TAG was observed in the $6 \mathrm{C}+4 \mathrm{CP}$ group. Thus, we conclude that the reduction of blood adiponectin level by $\mathrm{CP}$ ingestion does not lead to obesity.

During protein synthesis, unfolded proteins can accumulate in the ER and generate cellular stress, and the UPR system limits the effects of this type of cellular stress ${ }^{(41,42)}$. In the present study, the expressions of IRE1 and its target genes were down-regulated in the $6 \mathrm{C}+4 \mathrm{CP}$ group in comparison with the $10 \mathrm{C}$ group (Table 4 ). p-IRE1 functions as an RNase, splicing the mRNA of X-box-binding protein $(X b p 1)$, a key UPR transcription factor, to produce its active form. The genes induced by ER-stress sensing by IRE1 include 
Table 5. A list of Kyoto Encyclopedia of Genes and Genomes (KEGG) pathways and their component genes significantly up-regulated (false-discovery rate-corrected $P$-value $<0.05$ ) by collagen peptide ingestion

\begin{tabular}{|c|c|c|c|}
\hline KEGG pathways (KEGG-ID) & Gene title & Gene symbol & Rank \\
\hline \multicolumn{4}{|c|}{ Biosynthesis of unsaturated fatty acids (mmu01040) } \\
\hline & Acyl-CoA thioesterase 3 & Acot3 & 2 \\
\hline & Acyl-CoA thioesterase 2 & Acot2 & 13 \\
\hline & Acyl-CoA thioesterase 4 & Acot4 & 21 \\
\hline & YOD1 OTU deubiquitinating enzyme 1 homologue (S. cerevisiae) & Yod1 & 54 \\
\hline & Acetyl-CoA acyltransferase $1 A$ & Acaa1a & 59 \\
\hline & Stearoyl-CoA desaturase 1 & Scd1 & 79 \\
\hline & Acyl-CoA oxidase 1, palmitoyl & Acox 1 & 148 \\
\hline & Fatty acid desaturase 2 & Fads2 & 277 \\
\hline & Fatty acid desaturase 1 & Fads1 & 340 \\
\hline & Elongation of very long chain fatty acids (FEN1/Elo2, SUR4/Elo3, yeast)-like 2 & Elovl2 & 471 \\
\hline \multicolumn{4}{|c|}{ PPAR signaling pathway (mmu03320) } \\
\hline & Cytochrome P450, family 4, subfamily a, polypeptide 14 & Cyp4a14 & 4 \\
\hline & Cytochrome P450, family 4, subfamily a, polypeptide 10 & Cyp4a10 & 7 \\
\hline & Acyl-CoA synthetase long-chain family member 1 & Acsl1 & 18 \\
\hline & Malic enzyme 1, NADP(+)-dependent, cytosolic & Me1 & 27 \\
\hline & Glycerol kinase & Gk & 32 \\
\hline & Acetyl-CoA acyltransferase $1 A$ & Acaa1a & 59 \\
\hline & Enoyl-CoA, hydratase/3-hydroxyacyl CoA dehydrogenase & Ehhadh & 66 \\
\hline & Stearoyl-CoA desaturase 1 & Scd1 & 79 \\
\hline & Sorbin and $\mathrm{SH} 3$ domain containing 1 & Sorbs1 & 81 \\
\hline & Acyl-CoA oxidase 1, palmitoyl & Acox 1 & 148 \\
\hline & Carnitine palmitoyltransferase 1 a, liver & Cpt1a & 193 \\
\hline & Fatty acid desaturase 2 & Fads2 & 277 \\
\hline & Peroxisome proliferator-activated receptor alpha & Ppara & 349 \\
\hline & Solute carrier family 27 (fatty acid transporter), member 2 & Slc27a2 & 468 \\
\hline \multicolumn{4}{|c|}{ Fatty acid metabolism (mmu00071) } \\
\hline & Cytochrome P450, family 4, subfamily a, polypeptide 14 & Cyp4a14 & 4 \\
\hline & Cytochrome P450, family 4, subfamily a, polypeptide 10 & Сур4а10 & 7 \\
\hline & Acyl-CoA synthetase long-chain family member 1 & Acs/1 & 18 \\
\hline & Acetyl-Co $A$ acyltransferase $1 A$ & Acaa1a & 59 \\
\hline & Enoyl-CoA, hydratase/3-hydroxyacyl CoA dehydrogenase & Ehhadh & 66 \\
\hline & Aldehyde dehydrogenase family 3 , subfamily A2 & Aldh3a2 & 111 \\
\hline & Acyl-CoA oxidase 1, palmitoyl & Acox1 & 148 \\
\hline & Enoyl-Coenzyme A delta isomerase 2 & Eci2 & 162 \\
\hline & $\begin{array}{l}\text { Hydroxyacyl-CoA dehydrogenase/3-ketoacyl-CoA thiolase/enoyl-CoA } \\
\text { hydratase (trifunctional protein), beta subunit }\end{array}$ & Hadhb & 175 \\
\hline & Carnitine palmitoyltransferase 1a, liver & Cpt1a & 193 \\
\hline
\end{tabular}

those encoding proteases and chaperones, which function to reduce levels of accumulated proteins ${ }^{(43-45)}$. A direct search of the microarray data revealed that expression of the $X b p 1$ gene was also down-regulated in the $6 \mathrm{C}+4 \mathrm{CP}$ group (rank: 149, 271 of the 395 significantly down-regulated probe sets, online Supplementary Table S1). Taken together, these results suggest that ER stress is suppressed by CP ingestion, although the possibility of general reduction in protein synthesis requires further examination. Collagen contains the unique amino acid hydroxyproline (Hyp), and it was reported that ingested CP is digested and absorbed in part as Hyp-containing oligopeptides, such as prolylhydroxyproline (Pro-Hyp) or hydroxyprolylglycine (Hyp-Gly) ${ }^{(46-49)}$. The blood concentration of Pro-Hyp can reach approximately $50 \mu \mathrm{m}$ by $1-2 \mathrm{~h}$ after ingestion of $25 \mathrm{~g}$ of $\mathrm{CP}^{(50)}$. Pro-Hyp exhibits physiological activity against cultured skin fibroblasts ${ }^{(51)}$ and pre-adipocytes $^{(52)}$, whereas Hyp-Gly modulates the differentiation of cartilage cells in vitro and in vivo ${ }^{(53)}$. These data suggest that collagen-derived oligopeptides are responsible, at least in part, for the beneficial in vivo effects of collagen ingestion. Although the precise mechanisms underlying the modulation of cell function by collagen oligopeptides are currently unclear, it seems possible that they are incorporated into cells through the proton-coupled peptide transporters 1 and 2 (PEPT1 and PEPT2) and peptide/histidine transporters 1 and 2 (PHT1 and PHT2 or Ci1 in mice), the genes of which are expressed in Pro-Hypresponsive cells ${ }^{(52)}$. In this context, it is noteworthy that protoncoupled peptide transporter genes are expressed in the liver ${ }^{(54)}$.

In conclusion, the results of the present study focusing on the effects of collagen ingestion on liver gene expression reveal that ingested $\mathrm{CP}$ alters the expressions of genes related to lipid metabolism. Moreover, the blood cholesterol-lowering effect of $\mathrm{CP}$ ingestion was reproduced in the present study, and it was presumed that the effect is not due to enhancement of primary bile acid biosynthesis according to DNA microarray analysis of the liver. Although the precise mechanisms remain unclear, novel functions of collagen in the broader metabolic pathways of the liver are predicted on the basis of the decreased expression of genes related to the UPR.

\section{Acknowledgements}

The authors are grateful to Professor Hisanori Kato and Dr Lila Otani (The University of Tokyo) for meaningful discussions regarding this study. 
This research received no specific grant from any funding agency or from commercial or not-for-profit sectors.

Conceived and designed the experiments: C. T., Y.-i. K., K. T., K. A. and Y. N. Performed the experiments: C. T., Y.-i. K., T. I., T. T., M. T. and K. T. Analysed the data: C. T. and Y. N. Wrote the paper: C. T., Y.-i. K. and Y. N.

The authors declare that there are no conflicts of interest.

\section{Supplementary material}

For supplementary material/s referred to in this article, please visit https://doi.org/10.1017/S0007114516004384

\section{References}

1. Eastoe JE (1967) Composition of collagen and allied proteins. In Treaties on Collagen, vol. 1, pp. 1-72 [GN Ramachandran, editor]. London: Academic Press.

2. Koyama Y, Hirota A, Mori H, et al. (2001) Ingestion of gelatin has differential effect on bone mineral density and body weight in protein undernutrition. J Nutr Sci Vitaminol (Tokyo) 47, 84-86.

3. Wu J, Fujioka M, Sugimoto K, et al. (2004) Assessment of effectiveness of oral administration of collagen peptide on bone metabolism in growing and mature rats. J Bone Miner Metab 22, 547-553.

4. Zdzieblik D, Oesser S, Baumstark MW, et al. (2015) Collagen peptide supplementation in combination with resistance training improves body composition and increases muscle strength in elderly sarcopenic men: a randomised controlled trial. Br J Nutr 114, 1237-1245.

5. Walrand S, Chiotelli E, Noirt F, et al. (2008) Consumption of a functional fermented milk containing collagen hydrolysate improves the concentration of collagen-specific amino acids in plasma. J Agric Food Chem 56, 7790-7795.

6. Schauss AG, Stenehjem J, Park J, et al. (2012) Effect of the novel low molecular weight hydrolyzed chicken sternal cartilage extract, BioCell Collagen, on improving osteoarthritis-related symptoms: a randomized, double-blind, placebo-controlled trial. J Agric Food Chem 60, 4096-4101.

7. Minaguchi J, Koyama Y, Meguri N, et al. (2005) Effects of ingestion of collagen peptide on collagen fibrils and glycosaminoglycans in Achilles tendon. J Nutr Sci Vitaminol (Tokyo) 51, 169-174.

8. Matsuda N, Koyama Y, Hosaka Y, et al. (2006) Effects of ingestion of collagen peptide on collagen fibrils and glycosaminoglycans in the dermis. J Nutr Sci Vitaminol (Tokyo) 52, 211-215.

9. Saiga A, Iwai K, Hayakawa $\mathrm{T}$, et al. (2008) Angiotensin I-converting enzyme-inhibitory peptides obtained from chicken collagen hydrolysate. J Agric Food Chem 56, 9586-9591.

10. Herregods G, Van Camp J, Morel N, et al. (2011) Angiotensin I-converting enzyme inhibitory activity of gelatin hydrolysates and identification of bioactive peptides. J Agric Food Chem 59, 552-558.

11. Ratnayake WM, Sarwar G \& Laffey P (1997) Influence of dietary protein and fat on serum lipids and metabolism of essential fatty acids in rats. Br J Nutr 78, 459-467.

12. Lin B, Zhang F, Yu Y, et al. (2012) Marine collagen peptides protect against early alcoholic liver injury in rats. $\mathrm{Br} J \mathrm{Nutr}$ 107, 1160-1166.

13. Oliveira DR, Portugal LR, Cara DC, et al. (2001) Gelatin intake increases the atheroma formation in apoE knock out mice. Atherosclerosis 154, 71-77.
14. Koyama Y \& Kusubata M (2013) Effects of collagen peptide ingestion on blood lipids in rats fed a high-lipid and high-sucrose diet. Food Sci Technol Res 19, 1149-1153.

15. Terpstra AH, Hermus RJ \& West CE (1983) The role of dietary protein in cholesterol metabolism. World Rev Nutr Diet $\mathbf{4 2}$, $1-55$.

16. Tsung A, Geller DA, Colnot S, et al. (2011) Part I, liver cells and functions. In Molecular Pathology of Liver Diseases, Molecular Pathology Library 5, pp. 1-180 [SP Monga, editor]. New York: Springer.

17. Hochreiter S, Clevert DA \& Obermayer K (2006) A new summarization method for Affymetrix probe level data. Bioinformatics 22, 943-949.

18. $\mathrm{R}$ Development Core Team (2008) $R$ : A Language and Environment for Statistical Computing. Vienna: R Foundation for Statistical Computing.

19. Gentleman RC, Carey VJ, Bates DM, et al. (2004) Bioconductor: open software development for computational biology and bioinformatics. Genome Biol 5, R80.

20. Breitling R, Armengaud P, Amtmann A, et al. (2004) Rank products: a simple, yet powerful, new method to detect differentially regulated genes in replicated microarray experiments. FEBS Lett 573, 83-92.

21. Huang da W, Sherman BT \& Lempicki RA (2009) Systematic and integrative analysis of large gene lists using DAVID bioinformatics resources. Nat Protoc 4, 44-57.

22. Hosack DA, Dennis G Jr, Sherman BT, et al. (2003) Identifying biological themes within lists of genes with EASE. Genome Biol 4, R70.

23. Benjamini Y \& Hochberg Y (1995) Controlling the false discovery rate: a practical and powerful approach to multiple testing. J R Stat Soc Ser B 57, 289-300.

24. Pyper SR, Viswakarma N, Yu S, et al. (2010) PPARo: energy combustion, hypolipidemia, inflammation and cancer. $\mathrm{NuCl}$ Recept Signal 8, e002.

25. Grygiel-Górniak B (2014) Peroxisome proliferator-activated receptors and their ligands: nutritional and clinical implications - a review. Nutr J 13, 17.

26. Pawlak M, Lefebvre P \& Staels B (2015) Molecular mechanism of PPAR $\alpha$ action and its impact on lipid metabolism, inflammation and fibrosis in non-alcoholic fatty liver disease. J Hepatol 62 , 720-733.

27. Mezei O, Banz WJ, Steger RW, et al. (2003) Soy isoflavones exert antidiabetic and hypolipidemic effects through the PPAR pathways in obese Zucker rats and murine RAW 264.7 cells. J Nutr 133, 1238-1243.

28. Inoue $\mathrm{H}$, Jiang XF, Katayama $\mathrm{T}$, et al. (2003) Brain protection by resveratrol and fenofibrate against stroke requires peroxisome proliferator-activated receptor alpha in mice. Neurosci Lett 352, 203-206.

29. Yajima H, Ikeshima E, Shiraki M, et al. (2004) Isohumulones, bitter acids derived from hops, activate both peroxisome proliferator-activated receptor alpha and gamma and reduce insulin resistance. J Biol Chem 279, 33456-33462.

30. Han KL, Choi JS, Lee JY, et al. (2008) Therapeutic potential of peroxisome proliferators-activated receptor-alpha/gamma dual agonist with alleviation of endoplasmic reticulum stress for the treatment of diabetes. Diabetes 57, 737-745.

31. Tan S, Gao B, Tao Y, et al. (2014) Antiobese effects of capsaicin-chitosan microsphere (CCMS) in obese rats induced by high fat diet. J Agric Food Chem 62, 1866-1874.

32. Purushotham A, Schug TT, Xu Q, et al. (2009) Hepatocyte-specific deletion of SIRT1 alters fatty acid metabolism and results in hepatic steatosis and inflammation. Cell Metab 9, 327-338.

33. Moreno M, Lombardi A, Silvestri E, et al. (2010) PPARs: nuclear receptors controlled by, and controlling, nutrient 
handling through nuclear and cytosolic signaling. PPAR Res, 2010 Article ID 435689.

34. Yan S, Yang XF, Liu HL, et al. (2015) Long-chain acyl-CoA synthetase in fatty acid metabolism involved in liver and other diseases: an update. World J Gastroenterol 21, 3492-3498.

35. Forman BM, Chen J \& Evans RM (1997) Hypolipidemic drugs, polyunsaturated fatty acids, and eicosanoids are ligands for peroxisome proliferator-activated receptors alpha and delta. Proc Natl Acad Sci U S A 94, 4312-4317.

36. Nagaoka S, Awano T, Nagata N, et al. (1997) Serum cholesterol reduction and cholesterol absorption inhibition in $\mathrm{CaCo}-$ 2 cells by a soyprotein peptic hydrolyzate. Biosci Biotechnol Biochem 61, 354-356.

37. Nagaoka S, Futamura Y, Miwa K, et al. (2001) Identification of novel hypocholesterolemic peptides derived from bovine milk beta-lactoglobulin. Biochem Biophys Res Commun 281, 11-17.

38. Wang L, Lee YK, Bundman D, et al. (2002) Redundant pathways for negative feedback regulation of bile acid production. Dev Cell 2, 721-731.

39. Matsuzawa Y, Funahashi T, Kihara S, et al. (2004) Adiponectin and metabolic syndrome. Arterioscler Thromb Vasc Biol 24, 29-33.

40. Fisman EZ \& Tenenbaum A (2014) Adiponectin: a manifold therapeutic target for metabolic syndrome, diabetes, and coronary disease? Cardiovasc Diabetol 13, 103.

41. Ron D \& Walter P (2007) Signal integration in the endoplasmic reticulum unfolded protein response. Nat Rev Mol Cell Biol 8, 519-529.

42. Jo H, Choe SS, Shin KC, et al. (2013) Endoplasmic reticulum stress induces hepatic steatosis via increased expression of the hepatic very low-density lipoprotein receptor. Hepatology 57, 1366-1377.

43. Kaneko M, Ishiguro M, Niinuma $\mathrm{Y}$, et al. (2002) Human HRD1 protects against ER stress-induced apoptosis through ER-associated degradation. FEBS Lett 532, 147-152.

44. Lee AH, Iwakoshi NN \& Glimcher LH (2003) XBP-1 regulates a subset of endoplasmic reticulum resident chaperone genes in the unfolded protein response. Mol Cell Biol 23, 7448-7459.

45. Oda Y, Okada T, Yoshida H, et al. (2006) Derlin-2 and Derlin-3 are regulated by the mammalian unfolded protein response and are required for ER-associated degradation. J Cell Biol $\mathbf{1 7 2}$ 383-393.

46. Iwai K, Hasegawa T, Taguchi Y, et al. (2005) Identification of food-derived collagen peptides in human blood after oral ingestion of gelatin hydrolysates. J Agric Food Chem $\mathbf{5 3}$, 6531-6536.

47. Ohara H, Matsumoto H, Ito K, et al. (2007) Comparison of quantity and structures of hydroxyproline-containing peptides in human blood after oral ingestion of gelatin hydrolysates from different sources. J Agric Food Chem 55, 1532-1535.

48. Ichikawa S, Morifuji M, Ohara H, et al. (2010) Hydroxyprolinecontaining dipeptides and tripeptides quantified at high concentration in human blood after oral administration of gelatin hydrolysate. Int J Food Sci Nutr 61, 52-60.

49. Shigemura Y, Akaba S, Kawashima E, et al. (2011) Identification of a novel food-derived collagen peptide, hydroxyprolyl-glycine, in human peripheral blood by pre-column derivatisation with phenyl isothiocyanate. Food Chem 129, 1019-1024

50. Taga Y, Kusubata M, Ogawa-Goto K, et al. (2014) Highly accurate quantification of hydroxyproline-containing peptides in blood using a protease digest of stable isotope-labeled collagen. J Agric Food Chem 62, 12096-12102.

51. Shigemura Y, Iwai K, Morimatsu F, et al. (2009) Effect of prolyl-hydroxyproline (Pro-Hyp), a food-derived collagen peptide in human blood, on growth of fibroblasts from mouse skin. J Agric Food Chem 57, 444-449.

52. Minaguchi J, Tometsuka C, Koyama Y, et al. (2012) Effects of collagen-derived oligopeptide prolylhydroxyproline on differentiation of mouse 3T3-L1 preadipocytes. Food Sci Technol Res 18, 593-599.

53. Nakatani S, Mano H, Sampei C, et al. (2009) Chondroprotective effect of the bioactive peptide prolyl-hydroxyproline in mouse articular cartilage in vitro and in vivo. Osteoarthritis Cartilage 17, 1620-1627.

54. Smith DE, Clémençon B \& Hediger MA (2013) Proton-coupled oligopeptide transporter family SLC15: physiological, pharmacological and pathological implications. Mol Aspects Med 34, 323-336. 